\title{
Vibrio alginolyticus-associated wound infection acquired in British waters, Guernsey, July 2011
}

G D Reilly¹, C A Reilly², E G Smith³, C Baker-Austin (craig.baker-austin@cefas.co.uk)4

1. Department of Dermatology, Princess Elizabeth Hospital, Guernsey

2. Barts and The London Medical School, London, United Kingdom

3. Health Protection Agency, Public Health Laboratory, Birmingham, West Midlands, United Kingdom

4. Centre for Environment Fisheries and Aquaculture Science, Weymouth, Dorset, United Kingdom

Reilly GD, Reilly CA, Smith EG, Baker-Austin C. Vibrio alginolyticus-associated wound infection acquired in British waters, Guernsey, July 2011. Euro Surveill. 2011;16(42):pii=19994. Available online: http://www.eurosurveillance.org/ViewArticle.aspx?Articleld=19994

Article published on 20 October 2011

In July 2011, a Vibrio alginolyticus infection was diagnosed in a woman from Guernsey in the Channel Islands, British Isles after sea bathing and application of a natural seaweed dressing to a pre-existing leg wound. Microbiological investigation confirmed Vibrio in the wound and the species of seaweed used for the dressing. The washing of open wounds in seawater and use of unsterilised seaweed dressings should be discouraged, particularly in individuals with underlying risk conditions.

\section{Background}

Vibrio alginolyticus is a halophilic (salt-tolerant) Gramnegative bacterium found naturally in temperate marine and estuarine environments. This species is recognised as a human pathogen, and the incidence of infection significantly increases during summer months [1]. V. alginolyticus is ubiquitous in seawater and tends to cause superficial wound and ear infections (otitis media and otitis externa) [2]. Most reports of $V$. alginolyticus wound infections result from exposure of cuts or abrasions to contaminated seawater. V. alginolyticusassociated infections may be resolved using appropriate antibiotics, however, very rarely these infections can progress to bacteraemia and necrotising fasciitis, particularly in the immunocompromised.

\section{Case report}

In July 2011, a woman in her 70 s presented to the dermatology clinic in Guernsey in the Channel Isles, British Isles, with a non-healing infected wound on her lower leg. The patient was not receiving any medication, was otherwise healthy and had no past history of diabetes or other chronic conditions. Two weeks previously the patient had injured her leg on a plant pot in the garden. The patient continued her habit of swimming regularly in the sea off Guernsey and also cleaned the wound with a salt water solution made up at home and applied a seaweed dressing. The patient used a gel dressing which she had extracted from the receptacles of spiral wrack (Fucus spiralis), a seaweed collected locally from the beach at low tide, which was used to cover the wound under cling film. After two or three days the wound scabbed over and was left to heal. Ten days later the scab fell off whilst swimming in the sea. The wound reopened and she reapplied the dressing as before using seaweed gel under cling film for a further three days. As the wound was failing to heal, the patient presented to the clinic. On examination crusting erythema surrounding the wound was noted, indicative of an infection (Figure).

\section{Laboratory analysis}

A charcoal swab was taken for bacteriological culture, and this resulted in a pure growth of a Gram-negative bacterium. Based on colony morphology, tolerance to $\mathrm{NaCl}$ and case history, a pathogenic marine Vibrio was suspected, and a preliminary identification of $V$. alginolyticus was made after biochemical analysis using API 20E (Biomerieux, Marcy l'Etoile, France). The patient was treated with doxycycline $(100 \mathrm{mg}$ twice daily) for two weeks. At a subsequent follow up three weeks later the wound was completely healed.

\section{FIGURE}

Post-exposure aspect of leg wound, with crusting and surrounding erythema showing evidence of infection, Guernsey, June 2011

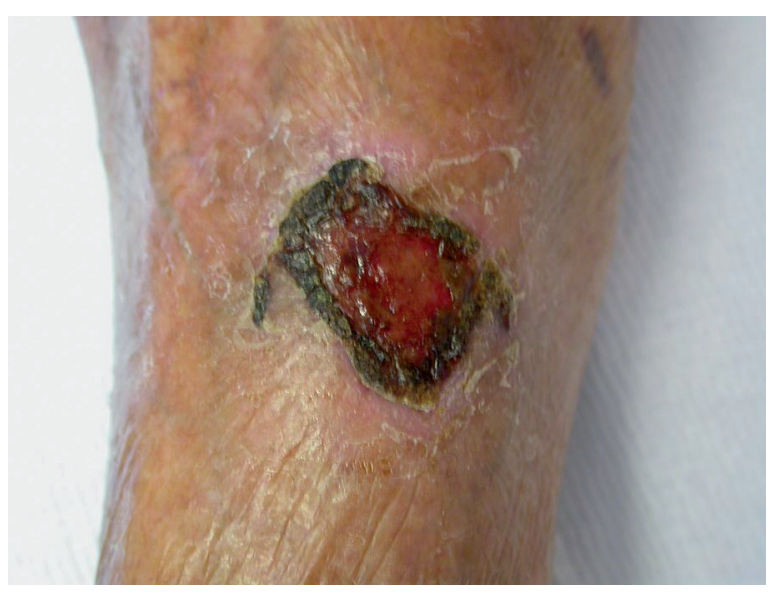


Microbiological analysis was subsequently performed on the isolated bacterial strain encompassing culturebased, molecular, and biochemical testing for unambiguous identification and characterisation. The bacterial cultures were subcultured on marine agar, thiosulfate citrate bile sucrose (TCBS) agar and chromID Vibrio agar (Biomerieux, Marcy l'Etoile, France) overnight at $30{ }^{\circ} \mathrm{C}$ and $37^{\circ} \mathrm{C}$. The distinctive morphology and colour of isolated colonies (yellow/orange on TCBS and chromID agar and cream colouration on marine agar), coupled to biochemical analysis $(97.8 \%$ species-level confirmation), were indicative of $V$. alginolyticus. Ten single colonies of presumptive $V$. alginolyticus strains from each set of agar plates were subsequently analysed by PCR, using primers recognising two separate $V$. alginolyticus species-specific targets, collegenase and the DNA replication gene gyrB, essentially as previously described $[2,4]$. In both cases, positive results were obtained confirming that the bacterium isolated was a $V$. alginolyticus strain.

Analysis of the seaweed and seawater samples obtained from the beach where the patient had bathed was also performed. Seaweed samples $(25 \mathrm{~g}$ mechanically disrupted in a laboratory blender with $25 \mathrm{ml}$ of alkaline saline peptone water) were found positive for $V$. alginolyticus by $\mathrm{PCR}$, however no $V$. alginolyticus strains were identified in analysed water.

\section{Discussion and conclusions}

V. alginolyticus wound infections are rare in Europe, with sporadic cases previously reported in the UK [5], the Netherlands [6] and Denmark [7]. Strikingly, as with other pathogenic Vibrio species, cases appear to be correlated with warm surface seawater temperatures, and it has been suggested that the number of infections may increase with warming of coastal regions attributed to climate change [8]. Although the summer of 2011 was not anomalously warm by recent standards, the infection corresponded with peak surface seawater temperatures (circa $18{ }^{\circ} \mathrm{C}$ ) experienced at the end of June/beginning of July 2011. It is striking that these infections are now beginning to be reported in temperate and 'non-endemic' regions, such as in European waters. To our knowledge, this is the first Vibrio wound infection reported in the UK in over 20 years. Recent reports have suggested that the number of bathing water-associated Vibrio cases in northern Europe are increasing [8], and other studies in Europe have reported Vibrio wound infections acquired through the intentional washing of wounds in seawater [9]. Previous work has suggested that alginate gels derived from seaweed are highly absorbent and biodegradable dressings that can be successfully applied to cleanse a wide variety of secreting lesions, facilitating healing [10]. To date, little reliable data is available to demonstrate the safety of home-prepared alginate dressings used in this context. Here the dressing used probably represented an effective route of exposure of an open wound to pathogenic Vibrio species, initiating infection. Although the patient described here did not have underlying medical conditions, this report highlights the potential health risks associated with the practice of using of non-sterile seawater and seaweed for wound cleansing purposes. Given the recent increase in reports of more serious Vibrio wound infections in northern Europe [8], including cases associated with $V$. vulnificus and non- $0_{1} V$. cholerae, this practise should be discouraged, particularly in vulnerable groups such as the elderly and individuals with underlying risk conditions. Medical Practitioners should be aware and consider marine Vibrio species as a possible cause of non healing wound infections in this group of patients.

Acknowledgments

We thank Paul Sutton and Andy Powell for technical assistance regarding bacterial isolation and culturing.

\section{References}

1. Morris JG Jr, Black, RE. Cholera and other vibrioses in the United States. N Engl J Med. 1985;312(6):343-50.

2. Pezzlo M, Valter PJ, Burns MJ. Wound infection associated with Vibrio alginolyticus. Am J Clin Pathol. 1979;71(4):476-8.

3. Di Pinto A, Ciccarese G, Tantillo G, Catalano D, Forte VT. A collagenase-targeted multiplex PCR assay for identification of Vibrio alginolyticus, Vibrio cholerae, and Vibrio parahaemolyticus, J Food Prot. 2005;68(1):150-3.

4. Luo P, Hu C. Vibrio alginolyticus gyrB sequence analysis and gyrB-targeted PCR identification in environmental isolates. Dis Aquat Organ. 2008;82(3):209-16.

5. Hartley JW, West E, Gothard WP, Hanan HW. Vibrio alginolyticus in the UK. J Infect. 1991;23(2):223.

6. Schets FM, de Roda Husman AM, Havelaar AH. Disease outbreaks associated with untreated recreational water use. Epidemiol Infect. 2010;10:1-12.

7. Holt H, Christensen JJ, Bruun B, Glismann S. Infections with seawater bacteria. EPI-NEWS. 2006;26-32. Available from: http://www.ssi.dk/English/News/EPI-NEWS/ /media/Indhold/ EN\%20-\%20engelsk/EPI-NEWS/2006/PDF/EPI-NEWS\%20-\%20 2006\%20-\%20No\%2026-32.ashx

8. Baker-Austin C, Stockley L, Rangdale R, Martinez-Urtaza J. Environmental occurrence and clinical impact of Vibrio vulnificus and Vibrio parahaemolyticus: a European perspective. Environ Microbiol Rep. 2010;2(1):7-18.

9. Lukinmaa S, Mattila, K Lehtinen L, Hakkinen M, Koskela M, Siitonen A. Territorial waters of the Baltic Sea as a source of infections caused by Vibrio cholerae non- 01 , non- 0139 report of 3 hospitalized cases. Diagn Microbiol Infect Dis. 2006;54(1):1-6.

10. Gilchrist T, Martin AM., Wound treatment with Sorbsan - an alginate fibre dressing. Biomaterials. 1983;4(4):317-20. 